\title{
Analysis of Circulating System Frictional Pressure Loss in Microhole Drilling with Coiled Tubing
}

\author{
Hou Xuejun ${ }^{1,2, *}$, Zheng Huikai ${ }^{3}$, Zhao Jian ${ }^{4}$ and Chen Xiaochun ${ }^{4}$
}

\begin{abstract}
${ }^{1}$ College of Petrol Engineering, Chongqing University of Science and Technology, Chongqing 401331, China; ${ }^{2}$ Harold Vance Department of Petroleum Engineering, Texas A\&M University, College Station, TX, 77840, USA, ${ }^{3}$ Tianjin Boxing Science and Technology Engineering Ltd, Offshore Oil Engineering Ltd. of PetroChina, Tianjin 300451, China; ${ }^{4}$ Engineering Department, Qingdao TaiNeng Gas Co. Ltd, Qingdao 266000, China
\end{abstract}

\begin{abstract}
In microhole drilling (MHD) with coiled tubing (CT), the calculation models of drilling fluid circulating frictional pressure loss (DFCFPL) are studied for different flow regimes of drilling fluid in CT on reel, downhole CT and annulus. Example analysis of DFCFPL for $89 \mathrm{~mm}$ diameter microhole is conducted, and the relationships among the total DFCFPL, local DFCFPL in CT on reel, downhole CT and annulus are obtained. The smaller the CT diameter is, the higher the local DFCFPL in CT on reel and downhole CT are. The larger the annulus is, the lower the local DFCFPL in annulus is. So the local DFCFPL in CT is dominant in the circulating system. As for the different diameter CT, the total DFCFPL decreases linearly with the well depth increasing. In order to use the hydraulic energy rationally, $73.025 \mathrm{~mm}$ diameter CT can be used to drill microhole with well depth less than $1000 \mathrm{~m}, 60.325 \mathrm{~mm}$ diameter CT should be used to drill microhole well depth of which is more than $1500 \mathrm{~m}$. This study can provide references for the CT selection and circulating hydraulic energy rational utilization design in MHD.
\end{abstract}

Keywords: Circulating frictional pressure loss, coiled tubing, drilling fluid, microhole drilling, power-law fluid.

\section{INTRODUCTION}

The microhole drilling (MHD) technology is one of the new frontier drilling technologies with wellbore diameter less than $88.9 \mathrm{~mm}$ and taking coiled tubing (CT) as drilling string to deliver the BHA to drill ahead [1]. It has many advantages, such as low cost, efficient, environmental protection, easy to achieve drilling informatization, automation and intelligentization [2], which lead to this technology [3]. Whereas, drilling fluid circulating frictional pressure loss (DFCFPL) in MHD is huge because of the small diameter wellbore, CT and narrow annulus interval. The total DFCFPL mainly consists of local DFCFPL in CT, local DFCFPL of drill bit and local DFCFPL in annulus, and DFCFPL in CT also consists of local DFCFPL in CT wound around reel and local DFCFPL in downhole CT. Lots of researches on DFCFPL in conventional CT downhole operation have been conducted by domestic and foreign scholars. In overseas, Ian.C [4] et al. devised the DFCFPL for CT drilling and R.C. McCann et al. [5] researched the DFCFPL of turbulent fluid in CT in 1996. In 1998, I. Azouz and S.N. Shah, P.S. [6] conducted the experiment studies on DFCFPL in CT. In 2000, B. Medjani and S.N. Shah [7] predicted the DFCFPL of non-Newtonian fluid in CT. J.D. Willingham and S. N. Shah [8] researched the DFCFPL of Newtonian fluid and non-Newtonian fluid in CT in reel and vertical well section. From 2001, Subhash N. [9, 10] studied the impact of

*Address correspondence to this author at the College of Petrol Engineering, Chongqing University of Science and Technology, Chongqing 401331, China; Tel: 023-65023083;

E-mails: xuejun_hou_2013@163.com and boxking2013@126.com drilling cuttings on DFCFPL and predicted the DFCFPL in CT fracturing process. B. N. Rao, Y. Zhou and S.N. Shah $[11,12]$ studied the impact factors of DFCFPL for nonNewtonian fluid in CT. M. B. Bailey [13] analyzed the fluid flow in CT injection nozzle on reel. In domestic, Ma Dongjun [14] studied the DFCFPL in coiled tubing ultra-short radius drilling process. Zhou Y [15] studied the DFCFPL of power-law fluid in $\mathrm{CT}$ by theoretical and experimental methods. Niu Tao [16] studied the DFCFPL in microhole circulating system. Whereas, few researches about total DFCFPL and local DFCFPL in CT on reel, in downhole CT and annulus have been reported for MHD so far. Thus, for different flow regimes of drilling fluid in $\mathrm{CT}$ on reel, downhole CT and annulus, the calculation models of DFCFPL are studied for MHD. The analysis can provide references for the parameters design and selection of $89 \mathrm{~mm}$ diameter MHD fluid circulation by analyzing the example of DFCFPL for $89 \mathrm{~mm}$ diameter microhole well and analyzing the variation regulation of the total DFCFPL and local DFCFPL comparatively.

\section{CALCULATION MODEL OF DFCFPL FOR POWER-LAW FLUID}

\subsection{Rheological Equation of Power-Law Fluid}

In conventional drilling process, the realistic rheological curve of drilling fluid is relatively familiar to power-law fluid. Thus, rheological model of power-law fluid is commonly used to calculate the DFCFPL in CT, its corresponding rheological equation, parameters and calculation models [17] are expressed as follows: 


$$
\left\{\begin{array}{l}
\tau=K\left(\frac{d u}{d y}\right)^{n} \\
n_{P}=3.322 \log _{10}\left(\frac{\phi_{600}}{\phi_{300}}\right) \& n_{A}=0.5 \log _{10}\left(\frac{\phi_{300}}{\phi_{3}}\right) \\
K_{P}=\frac{0.511 \phi_{600}}{1022^{n_{p}}} \& \quad K_{\mathrm{A}}=\frac{0.511 \phi_{000}}{511^{a_{a}}}
\end{array}\right.
$$

Where, $\phi_{600}, \phi_{300}$ and $\phi_{3}$ stand for the readings of rotary viscometer while its rotate speed is $600 \mathrm{r} / \mathrm{min}, 300 \mathrm{r} / \mathrm{min}$ and $3 \mathrm{r} / \mathrm{min}$ respectively; $K_{P}$ is the consistency coefficient of power-law fluid in CT and it is dependent on fluid property, dyn. $\mathrm{s}^{n} / \mathrm{cm}^{2} ; K_{A}$ is the consistency coefficient of powerlaw fluid in CT, dyn.s $\mathrm{s}^{n} / \mathrm{cm}^{2} ; n_{P}$ stands for the rheological index (liquidity index) of power-law fluid in CT, nondimensional, it represents the degree that the annular fluid deviates from Newtonian fluid; $n_{A}$ stands for the rheological index (liquidity index) of annulus power-law fluid; $\tau$ is shear stress, $P a$.

\subsection{Calculations of Reynolds Number}

Reynolds Number (Re) represents the ratio of inertia force and viscosity force, and it is the basis of judging fluid flow regimes. As for the power-law fluid in CT and annulus for CT MHD, the calculation equations of Re can be expressed as follows.

As for the fluid flow in CT, its Re can be expressed as follows:

$$
\operatorname{Re}_{p}=\frac{\rho d^{n_{P}} v_{P}{ }^{2-n_{P}}}{8^{n_{P}-1} K_{P}\left(\frac{3 n_{P}+1}{4 n_{P}}\right)^{n_{P}}}
$$

Where, $\operatorname{Re}_{P}$ stands for the Re of drilling fluid flowing in $\mathrm{CT}$, non-dimensional; $d$ stands for the inner diameter of CT, $\mathrm{m} ; \rho$ stands for the density of circulating drilling fluid in $\mathrm{CT}, \mathrm{kg} / \mathrm{m}^{3} ; v_{P}$ stands for the average flow rate of circulating drilling fluid in $\mathrm{CT}, \mathrm{m} / \mathrm{s}$; the other symbols have the same meanings as above.

As for the flow rate of drilling fluid in hole annulus, its Re can be expressed as follows:

$$
\operatorname{Re}_{A}=\frac{\rho\left(D_{h}-D_{0}\right)^{n_{A}} v_{A}{ }^{2-n_{A}}}{12^{n_{A}-1} K_{A}\left(\frac{2 n_{A}+1}{3 n_{A}}\right)^{n_{A}}}
$$

Where, $\operatorname{Re}_{A}$ stands for the Re of drilling fluid flowing in CT, non-dimensional; $v_{A}$ stands for the average flow rate of circulating drilling fluid in $\mathrm{CT}, \mathrm{m} / \mathrm{s} ; D_{h}$ stands for the diameter of wellbore, $\mathrm{m} ; D_{0}$ stands for the outer diameter of $\mathrm{CT}, \mathrm{m}$; the other symbols have the same meanings as above.
As for the CT twisting in the reel system in the MHD, CT reel diameter is so small that a long CT twisting in the reel system has a very small bending radius. While drilling fluid flows through the continuous curving CT, secondary Dean vortex will appear under the interaction of ever-changing viscous force and centrifugal force, which will cause a big impact on DFCFPL in CT on reel is much higher than the DFCFPL in straight section. W.R. Dean [18, 19] put forward the dimensionless dean number to measure the incidence of DFCFPL in CT on reel by studying the motion of fluid in a curved pipe.

$$
N_{D n}=\operatorname{Re}_{P} \times\left[\frac{r_{0}}{R_{i}}\right]^{0.5}
$$

Where, $N_{D n}$ is dean number, it is the ratio of the centrifugal force and viscous force, non-dimensional; $r_{0}$ stands for the radius of CT twisting in the reel system, $\mathrm{m} ; R_{i}$ stands for the radius of curvature for the No. $i$ layer CT which twists in the CT reel system, m; the other symbols have the same meanings as above.

\subsection{Flow Regimes Distinguish and Friction Factor Calculation}

Suppose the discriminant factors $C_{1}$ and $C_{2}$ are described in Eq. (5), by comparing Re with $C_{1}$ and $C_{2}$, different flow regimes of power-law fluid can be estimated and their corresponding Fanning friction factors [10] can be expressed as follows:

$$
\left\{\begin{array}{lll}
C_{1 P}=3470-1370 n_{P} & \& & C_{1 A}=3470-1370 n_{A} \\
C_{2 P}=4270-1370 n_{P} & \& & C_{2 A}=4270-1370 n_{A}
\end{array}\right.
$$

Where, $n$ stands for the liquidity index of power-law fluid, when the fluid is in hole annulus, $\mathrm{n}$ equals to $n_{A}$, while the fluid is in CT, $\mathrm{n}$ equals to $n_{P} ; C_{1 P}$ and $C_{2 P}$ are the discriminant factors of drilling fluid while it flows in CT respectively; $C_{1 A}$ and $C_{2 A}$ are the discriminant factors of drilling fluid while it flows in hole annulus respectively.

Based on the discriminant factors, flow regimes of drilling fluids in the circulation system in MHD can be distinguished and its corresponding calculation equations of Fanning friction factors can be described as follows:

(1) In the MHD circulating system, as for drilling fluid flowing in the CT (both in the surface reel and downhole), while $\operatorname{Re}_{P}<C_{1 P}$, the flow regime is laminar flow; as for drilling fluid flowing in the hole annulus, while $\operatorname{Re}_{A}<C_{1 A}$, the flow regime is laminar flow, its corresponding calculation equation of Fanning friction factors [20] can be described as follows:

$$
\left\{\begin{array}{l}
f_{P}=\frac{16}{\operatorname{Re}_{P}} \\
f_{A}=\frac{24}{\operatorname{Re}_{A}} \\
f_{r}=\frac{16}{N_{D n}}
\end{array}\right.
$$


Where, $f_{P}$ stands for the Fanning friction factor of drilling fluid flowing in the downhole $\mathrm{CT}$ while the flow regime is laminar flow, non-dimensional; $f_{A}$ stands for the Fanning friction factor of drilling fluid flowing in the wellbore annulus while the flow regime is laminar flow, non-dimensional; $f_{r}$ stands for the Fanning friction factor of drilling fluid flowing in the CT twisting in reel while the flow regime is laminar flow, non-dimensional; the other symbols have the same meanings as above.

(2) In the MHD circulating system, as for drilling fluid flowing in the CT (both in the surface reel and downhole), while $\operatorname{Re}_{P}>C_{2 P}$, the flow regime is turbulent flow; as for drilling fluid flowing in the hole annulus, while $\operatorname{Re}_{A}>C_{2 A}$, the flow regime is turbulent flow, its corresponding calculation equation of Fanning friction factors [20] can be described as follows:

$$
\left\{\begin{array}{l}
f_{P}=\frac{a_{P}}{\operatorname{Re}_{P}^{b_{P}}} \quad\left(a_{P}=\frac{\lg n_{P}+3.93}{50} \quad \& \quad b_{P}=\frac{1.75-\lg n_{P}}{7}\right) \\
f_{A}=\frac{a_{A}}{\operatorname{Re}_{A}^{b_{A}}} \quad\left(a_{A}=\frac{\lg n_{A}+3.93}{50} \quad \& \quad b_{A}=\frac{1.75-\lg n_{A}}{7}\right) \\
f_{r}=\frac{a_{P}}{N_{D n}^{b_{P}}}
\end{array}\right.
$$

Where, $f_{P}$ stands for the Fanning friction factor of drilling fluid flowing in the downhole CT while the flow regime is turbulent flow, non-dimensional; $f_{A}$ stands for the Fanning friction factor of drilling fluid flowing in the wellbore annulus while the flow regime is turbulent flow, nondimensional; $f_{r}$ stands for the Fanning friction factor of drilling fluid flowing in the $\mathrm{CT}$ twisting in reel while the flow regime is turbulent flow, non-dimensional; $a_{P} \& b_{P}$ are the calculation coefficients of drilling fluid flow resistance in CT respectively; $a_{A} \& b_{A}$ are the calculation coefficients of drilling fluid flow resistance in wellbore annulus respectively; the other symbols have the same meanings as above.

(3) In the MHD circulating system, as for drilling fluid flowing in the CT (both in the surface reel and downhole), while $C_{1 P} \leq \operatorname{Re}_{P} \leq C_{2 P}$, the flow regime is transitional flow; as for drilling fluid flowing in the hole annulus, while $C_{1 A} \leq \operatorname{Re}_{A} \leq C_{2 A}$, the flow regime is transitional flow, its corresponding calculation equation of Fanning friction factors [20] can be described as follows:

$$
\left\{\begin{array}{l}
f_{P}=\frac{16}{C_{1 P}}+\left(\frac{a_{P}}{C_{2 P}^{b_{P}}}+\frac{16}{C_{1 P}}\right) \frac{\mathrm{Re}_{P}-C_{1 P}}{800} \\
f_{A}=\frac{24}{C_{1 A}}+\left(\frac{a_{A}}{C_{2 A}^{b_{A}}}+\frac{24}{C_{1 A}}\right) \frac{\mathrm{Re}_{A}-C_{1 A}}{800} \\
f_{r}=\frac{16}{C_{1 P}}+\left(\frac{a_{P}}{C_{2 P}^{b_{P}}}+\frac{12}{C_{1 P}}\right) \frac{N_{D n}-C_{1 P}}{800}
\end{array}\right.
$$

Where, $f_{P}$ stands for the Fanning friction factor of drilling fluid flowing in the downhole $\mathrm{CT}$ while the flow regime is transitional flow, non-dimensional; $f_{A}$ stands for the Fanning friction factor of drilling fluid flowing in the wellbore annulus while the flow regime is transitional flow, nondimensional; $f_{r}$ stands for the Fanning friction factor of drilling fluid flowing in the CT twisting in reel while the flow regime is transitional flow, non-dimensional; the other symbols have the same meanings as above.

\subsection{Calculation Model of DFCFPL}

According to the Fanning equations, the calculation model of DFCFPL under different flow regimes in CT and annulus can be expressed as follows:

$$
\left\{\begin{array}{l}
\Delta p_{P}=\frac{2 \rho v_{p}^{2}}{d} f_{p} L_{P} \\
\Delta p_{A}=\frac{2 \rho v_{A}^{2}}{D_{h}-D_{0}} f_{A} L_{A} \\
\Delta p_{r}=\frac{2 \rho v_{r}^{2}}{d} \sum_{i=1}^{N} f_{r} L_{i}
\end{array}\right.
$$

Where, $\Delta p_{P}$ stands for the local DFCFPL in downhole $\mathrm{CT}$ while the flow regime is transitional flow, $\mathrm{MPa} ; \Delta p_{A}$ stands for the local DFCFPL in wellbore annulus while the flow regime is transitional flow, $\mathrm{MPa} ; \Delta p_{r}$ stands for the local DFCFPL in CT twisting on reel while the flow regime is transitional flow, $\mathrm{MPa} ; f_{p}, f_{A}$ and $f_{r}$ stand for the friction factors of drilling fluid flowing in the downhole CT, wellbore annulus and CT twisting on reel under different flow regimes respectively; $L_{P}$ is the length of downhole CT, $\mathrm{m} ; L_{A}$ is the length of wellbore annulus, $\mathrm{m} ; L_{i}$ stands for the length of the No. $i$ CT on reel, m; the other symbols have the same meanings as above.

\subsection{Calculation Model of Parameters of CT on Reel}

In the MHD, the local DFCFPL of surface pipeline mainly focuses on the CT twisting on reel. However, the DFCFPL with different CT parameters, such as different reel system and its different diameters, CT in different layers on the same reel has different length, bending radius and friction factor. A reel system CT simplified model is built for conventional analysis of the DFCFPL, shown in the (Fig. 1) as follows:

According to the reel system CT simplified model as shown in (Fig. 1), the calculation model of CT parameters is built as follows [21]:

$$
\left\{\begin{array}{l}
N=\left[\frac{A-E}{D}\right] \\
M=\left[\frac{C}{D}\right]=\left[\frac{C}{\left.2 r_{0}\right]}\right. \\
D_{\text {reel }}=B+2 A \\
R_{i}=\frac{\sqrt{\left[\left(2 r_{0}+B\right)+2 \sqrt{3}(i-1) r_{0}\right]^{2}+r_{0}^{2}}}{2} \quad(N \geq i \geq 1) \\
L=2 \pi M \sum_{i=1}^{N} R_{i} \quad \text { o } \quad L_{i}=2 \pi M R_{i}
\end{array}\right.
$$


Where, $N$ stands for the layer number of CT twisting in the reel system, integer; $A$ is the height of reel flange, m; $E$ is the distance from reel system outermost CT to reel system outer rim, $\mathrm{m} ; M$ is the column number of each layer CT twisting in the reel system, integer; $D$ is outer diameter of CT twisting in reel system, $\mathrm{m}$; $C$ is the rim width of CT reel system, $\mathrm{m} ; D_{\text {reel }}$ is the radius of CT reel, $\mathrm{m} ; B$ is the roller core diameter, $\mathrm{m}$; $L$ is the total length of CT twisting in the reel system, $\mathrm{m}$; the other symbols have the same meanings as above.

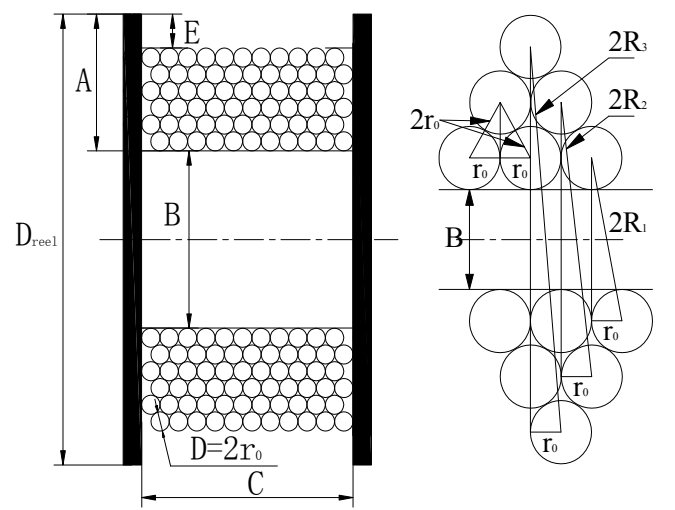

Fig. (1). Reel System CT String Simplified Analysis Model.

\section{EXAMPLE ANALYSIS OF DFCFPL IN MHD}

\subsection{Calculation Parameters Setting}

The CT twists in the roller core of the reel system, so it is easy to be flexural deformation, and the deformation is determined by CT size and roller core size. The general requirement of the diameter of reel system roller core is not less than 48 times of the $\mathrm{CT}$ diameter. According to the available CT can be used in the $89 \mathrm{~mm}$-diameter MHD, the selected CT with different diameter and its corresponding CT reels are listed as follows: the radius of CT reel is $4.06 \mathrm{~m}$, the height of reel flange is $0.61 \mathrm{~m}$, the roller core diameter is $2.845 \mathrm{~m}$, the rim width of CT reel system is $2.007 \mathrm{~m}$, the distance from reel system outermost CT to reel system outer $\operatorname{rim} E$ for different diameter CT are listed as (Table 1). Simultaneously, assume the density of low solid and low viscosity drilling fluid is $1.5 \mathrm{~g} / \mathrm{cm}^{3}, \phi_{600}$ is $35, \phi_{300}$ is 12 and $\phi_{3}$ is 2. The DFCFPL for $\varphi 89 \mathrm{~mm}$ MHD is calculated based on the equations $(1) \sim(10)$. The calculated resulting drawings are shown as follows.

\subsection{Contrastive Analysis of Example Analysis}

The total DFCFPL in MHD consists of local DFCFPL in CT twisting on reel, downhole CT, wellbore annulus and drill bit. The flow rate is constant in the circulation system if
DFCFPL of drill bit and exceptional circumstances such as lost circulation, overflow are not considered then cutting influence is neglected. Thus, suppose the flow rate of drilling fluid and length of CT are constant, the local DFCFPLs in CT on reel, downhole CT and wellbore annulus are calculated, and the results are drawn and analyzed as follows:

(1) The local DFCFPL in CT on reel, downhole CT and wellbore annulus increase quickly respectively with the flow rate increasing of drilling fluid as shown in (Fig. 2).

(2) While the CT length is constant, the smaller the CT inner diameter is, the higher the DFCFPLs in CT on reel and downhole CT are. The smaller the CT outer diameter is, the larger the annulus is, the lower the DFCFPL in annulus is. Therefore, the DFCFPL in CT is dominant in the circulating system. The larger the $\mathrm{CT}$ inner diameter is, the lower the DFCFPL in CT on reel and downhole CT are. The larger the CT outer diameter is, the smaller the annulus is, the higher the DFCFPL in annulus is. So the DFCFPL in annulus is dominant in the circulating system. In $\varphi 89 \mathrm{~mm}$ MHD, while the CT length is $2000 \mathrm{~m}$, the ratios of each local DFCFPL to the total DFCFPL are as follows: as for the $\varphi 44.45 \mathrm{~mm} \mathrm{CT}$, the ratio of DFCFPL in CT on reel to the global DFCFPL is about $60 \% \sim 61 \%$, the ratio of DFCFPL in downhohe $\mathrm{CT}$ is about $37 \% \sim 38 \%$, and the ratio of DFCFPL in wellbore annulus is the least, its value is $2.11 \% \sim 0.3 \%$ and can be neglected. As for the $50.8 \mathrm{~mm}$ diameter $\mathrm{CT}$, the ratio of DFCFPL in CT on reel to the global DFCFPL is about $57 \% \sim 60 \%$, the ratio of DFCFPL in downhohe CT is about $36 \% \sim 38 \%$, and the ratio of wellbore annulus is the least, its value is $6 \% \sim 1 \%$ and can be neglected. As for the $\varphi 60.325 \mathrm{~mm} \mathrm{CT}$, the ratio of DFCFPL in CT on reel to the total DFCFPL decrease to $47 \% \sim 57 \%$, the ratio of DFCFPL in downhohe CT is about 30\% 37\%, and the ratio of DFCFPL in wellbore annulus increases quickly to $22 \% \sim 5 \%$. As for the $\varphi 73.025 \mathrm{~mm} \mathrm{CT}$, the ratio of DFCFPL in CT on reel to the total DFCFPL decrease to $18 \% \sim 36 \%$, the ratio of DFCFPL in downhohe CT decreases to $12 \% \sim 23 \%$, and the ratio of DFCFPL in wellbore annulus increases quickly to $69 \% \sim 40 \%$ and exceeds the ratios of DFCFPL in CT on reel and downhole CT. The results are shown in (Table 2).

(3) While drilling the $\varphi 89 \mathrm{~mm}$ MHD with depth $5000 \mathrm{~m}$ by using 5000m CT drill rig, following the well depth increasing, the curves of total DFCFPL (Fig. 3) show that: while $25.4 \mathrm{~mm} \sim 60.325 \mathrm{~mm}$ diameter CT are used in the drilling process, the total DFCFPL decreases linearly with the well depth increasing, its value is the highest when $25.4 \mathrm{~mm}$ diameter CT is used, and is lowest with $60.325 \mathrm{~mm}$ diameter CT used. While $73.025 \mathrm{~mm}$ diameter $\mathrm{CT}$ is used in the MHD, the total DFCFPL increases

Table 1. Coiled tubing parameter list [22].

\begin{tabular}{|c|c|c|c|c|c|c|c|}
\hline Outer Diameter of CT(mm) & 25.4 & 31.750 & 38.100 & 44.450 & 50.800 & 60.325 & 73.025 \\
\hline Wall Thickness of CT(mm) & 3.175 & 3.962 & 3.962 & 3.962 & 3.962 & 3.962 & 3.962 \\
\hline Inner Diameter of CT(mm) & 19.05 & 23.826 & 30.176 & 36.526 & 42.876 & 52.401 & 65.101 \\
\hline $\mathrm{E}, \mathrm{mm}$ & 50.8 & 50.8 & 50.8 & 69.85 & 76.2 & 88.9 & 101.6 \\
\hline
\end{tabular}




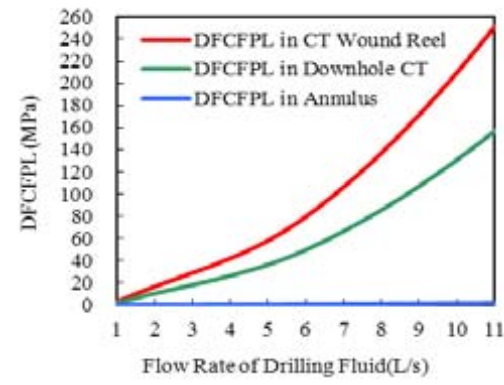

(a) $44.45 \mathrm{~mm}$ outer diameter CT

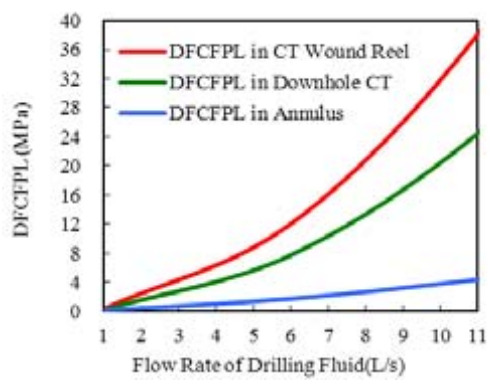

(c) $60.325 \mathrm{~mm}$ outer diameter CT

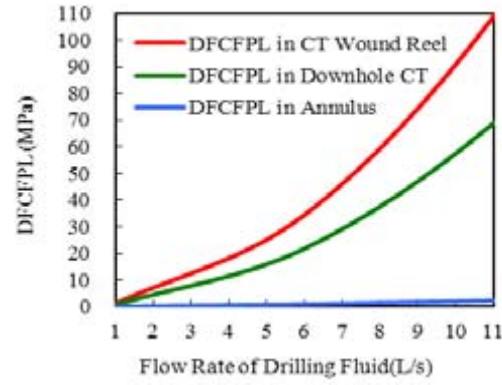

(b) $50.08 \mathrm{~mm}$ outer diameter CT

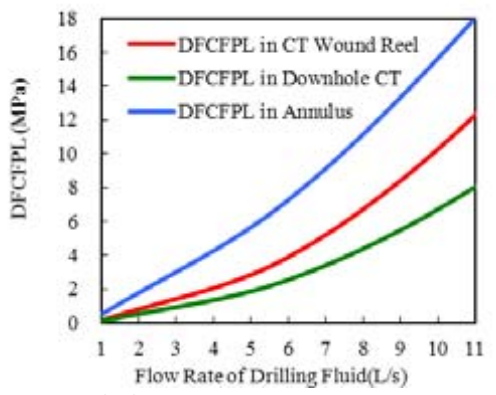

(d) $73.025 \mathrm{~mm}$ outer diameter CT

Fig. (2). DFCFPL Curves with Flow Rates in MHD with 2000m Length CT.

Table 2. The ratio of the local DFCFPL in CT on reel, downhole CT and annulus to the total DFCFPL for 2000m CT in MHD under different flow rates.

\begin{tabular}{|c|c|c|c|c|c|c|c|c|c|c|c|c|}
\hline \multirow{2}{*}{$\begin{array}{l}\text { Flow } \\
\text { Rate } \\
(\mathrm{L} / \mathbf{s})\end{array}$} & \multicolumn{3}{|c|}{$\begin{array}{c}\varphi 44.5 \mathrm{~mm} \mathrm{CT} \\
\varphi 89 \mathrm{~mm} \text { wellbore }\end{array}$} & \multicolumn{3}{|c|}{$\begin{array}{c}\varphi 50.8 \mathrm{~mm} \mathrm{CT} \\
\varphi 89 \mathrm{~mm} \text { wellbore }\end{array}$} & \multicolumn{3}{|c|}{$\begin{array}{c}\varphi 60.325 \mathrm{~mm} \mathrm{CT} \\
\varphi 89 \mathrm{~mm} \text { wellbore }\end{array}$} & \multicolumn{3}{|c|}{$\begin{array}{c}\varphi 73.025 \mathrm{~mm} \mathrm{CT} \\
\varphi 89 \mathrm{~mm} \text { wellbore }\end{array}$} \\
\hline & $\begin{array}{c}A \\
(\%)\end{array}$ & $\begin{array}{c}\text { B } \\
(\%)\end{array}$ & $\begin{array}{c}C \\
(\%)\end{array}$ & $\begin{array}{c}\mathrm{A} \\
(\%)\end{array}$ & $\begin{array}{c}\text { B } \\
(\%)\end{array}$ & $\begin{array}{c}\mathrm{C} \\
(\%)\end{array}$ & $\begin{array}{c}A \\
(\%)\end{array}$ & $\begin{array}{c}\text { B } \\
(\%)\end{array}$ & $\begin{array}{c}\mathrm{C} \\
(\%)\end{array}$ & $\begin{array}{c}\text { A } \\
(\%)\end{array}$ & $\begin{array}{c}\text { B } \\
(\%)\end{array}$ & $\begin{array}{c}C \\
(\%)\end{array}$ \\
\hline 1 & 60.26 & 37.63 & 2.11 & 57.58 & 36.42 & 6.00 & 47.06 & 30.25 & 22.69 & 18.37 & 12.01 & 69.62 \\
\hline 6 & 61.25 & 38.24 & 0.51 & 60.27 & 38.12 & 1.61 & 55.89 & 35.92 & 8.19 & 28.42 & 18.57 & 53.01 \\
\hline 11 & 61.31 & 38.28 & 0.40 & 60.48 & 38.25 & 1.27 & 56.88 & 36.56 & 6.56 & 32.04 & 20.93 & 47.03 \\
\hline 16 & 61.35 & 38.31 & 0.35 & 60.58 & 38.32 & 1.10 & 57.40 & 36.89 & 5.71 & 34.26 & 22.38 & 43.36 \\
\hline 21 & 61.37 & 38.32 & 0.31 & 60.65 & 38.36 & 0.99 & 57.74 & 37.11 & 5.16 & 35.84 & 23.42 & 40.74 \\
\hline
\end{tabular}

(Where, A is the ratio of the local DFCFPL in CT on reel to the total DFCFPL in MHD under different flow rates; B is the ratio of the local DFCFPL in downhole CT to the total DFCFPL in MHD under different flow rates; $\mathrm{C}$ is the ratio of the local DFCFPL in annulus CT to the total DFCFPL in MHD under different flow rates.)

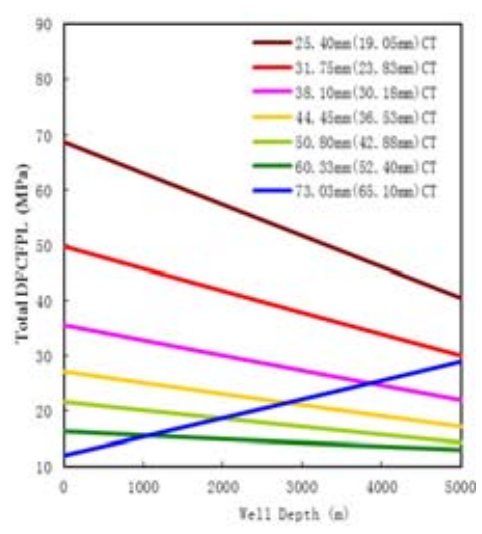

(a) $2 \mathrm{~m} / \mathrm{s}$ drilling fluid flow rate

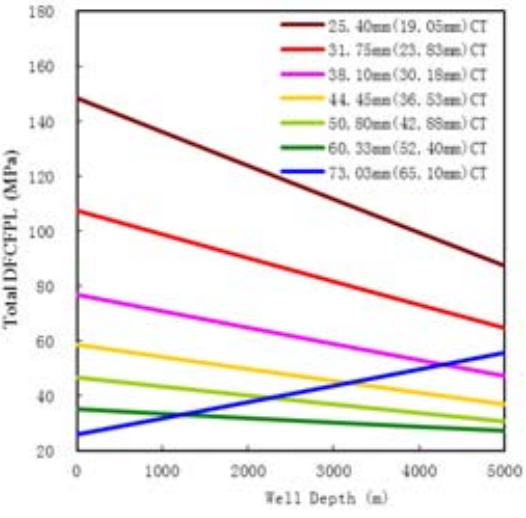

(b) $3 \mathrm{~m} / \mathrm{s}$ drilling fluid flow rate

Fig. (3). Total DFCFPL Curves With 5000m Well Depth in $89 \mathrm{~mm}$ Diameter MHD While Drilling Fluid Flow Rate is $2 \mathrm{~m} / \mathrm{s} \& 3 \mathrm{~m} / \mathrm{s}$ in CT. 
linearly with the well depth increasing, and its value exceeds the DFCFPL of $50.8 \mathrm{~mm}$ diameter CT. Thus, in order to use the hydraulic energy rationally, $73.025 \mathrm{~mm}$ diameter CT can be used to drill the well with depth less than $1000 \mathrm{~m}, 60.325 \mathrm{~mm}$ diameter $\mathrm{CT}$ should be used to drill the well whose depth is more than $1500 \mathrm{~m}$ to achieve the rational utilization of hydraulic energy.

(4) More reels can be used to decrease the DFCFPL in CT on reel in the MHD. When the CT on one reel is used up, the other can be connected to the circulating system. In this way, the length of CT on reel can be decreased in the MHD, so the DFCFPL in CT on reel can be decreased too.

(5) While the well depth is deep enough, the frictional force between CT and wellbore is huge because the CT string does not rotate, so draft gear should be used to drag the CT string up and in. If the draft gear is powered by circulating hydraulic energy, there should be differential pressure between inside and outside of bottom hole assembly. Thus, the local DFCFPL in CT cannot be too high so the proper functioning of draft gear can be guaranteed.

\section{CONCLUSIONS}

(1) The local DFCFPLs in CT on reel, downhole CT and wellbore annulus respectively increase quickly with the flow rate increasing. When the CT diameter is relatively large, the DFCFPL in annulus is dominant in the circulating system. When the CT diameter is small, the DFCFPL in CT is dominant in the circulating system.

(2) While the CT length is constant, the smaller the CT inner diameter is, the higher the DFCFPL in CT on reel and downhole $\mathrm{CT}$ are, and the smaller the CT outer diameter is, the larger the annulus is, the lower the DFCFPL in annulus is, so the DFCFPL in CT is dominant in the circulating system. The larger the $\mathrm{CT}$ inner diameter is, the lower the DFCFPL in CT on reel and downhole CT are, and the larger the CT outer diameter is, the smaller the annulus is, the higher the DFCFPL in annulus is, so the DFCFPL in annulus is dominant in the circulating system.

(3) For the $25.4 \mathrm{~mm} \sim 60.325 \mathrm{~mm}$ diameter CT used in the MHD with $5000 \mathrm{~m}$ well depth, the total DFCFPL decreases linearly with the well depth increasing. For the $73.025 \mathrm{~mm}$ diameter CT used in the MHD, the total DFCFPL increases linearly with the well depth increasing. Thus, in order to use the hydraulic energy rationally, $73.025 \mathrm{~mm}$ diameter $\mathrm{CT}$ can be used to drill less than $1000 \mathrm{~m}$ well depth, $60.325 \mathrm{~mm}$ diameter CT should be used to drill more than $1500 \mathrm{~m}$ well depth.

(4) More reels can be used to decrease the DFCFPL in CT on reel in the MHD. When the CT on one reel is used up, the other can be connected to the circulating system. In this way, the length of CT on reel can be decreased in the MHD, so the DFCFPL in CT on reel can be decreased and the total DFCFPL can be decreased too.

\section{CONFLICT OF INTEREST}

The authors confirm that this article content has no conflicts of interest.

\section{ACKNOWLEDGEMENTS}

The authors gratefully acknowledge the financial support of the Natural Science Foundation of China (NSFC,51374266), the Basic and Frontier Research Project by Chongqing Science and Technology Commission of China (Grant No.: cstc2013jcyjA90011), the Scientific and Technological Research Program of Chongqing Municipal Education Commission of China (Grant No.: KJ131413), the Key Cultivation Fund Projects (Grant No.: CK2013Z07), Scientific Research Funds Projects and Education Reform Project (Grant No.: 201140) of Chongqing University of Science \& Technology of China.

\section{REFERENCES}

[1] A. Jim, D. Don, A. Dave, and B. Jim, "Road Map for a 5000-ft microborehole", Los Alamos National Laboratory, pp.1-4, 2005.

[2] DOE, "DOE Announces R\&D funding for microhole technology projects", www.fossil.energy.gov, 2005.

[3] K. Perry, S. Bataresh, S. Gowelly, and T. Hayes, "Field demonstration of exiting microhole coiled tubing rig (MCTR) technology final technical report", Gas Technology Institute, pp.1-8, 2006.

[4] W. C. Ian, and H. Gu, "Hydraulics design in coiled tubing drilling", Society of Petroleum Engineers, vol. 36349, pp. 1-10, 1996.

[5] R.C. McCann, and C.G. Islas, "Frictional pressure loss during turbulent flow in coiled tubing", Society of Petroleum Engineers, vol. 36345, pp. 1-6, 1996.

[6] I. Azouz, S.N. Shah, P.S. Vinod, and D.L. Lord, "Experimental investigation of frictional pressure losses in coiled tubing", Society of Petroleum Engineers Production \& Facilities, vol. 13, pp. 91-96, 1998.

[7] B.Medjani, and S.N.Shah, "A new approach for predicting frictional pressure losses of non-newtonian fluid in coiled tubing", Society of Petroleum Engineers, vol. 60319, pp. 1-12, 2000.

[8] J. D. Willingham, and S. N. Shah, "Friction pressures of Newtonian and non-newtonian fluids in straight and reeled coiled tubing", Society of Petroleum Engineers, vol. 60719, pp. 1-13, 2000.

[9] S. N. Shah, and Y. Zhou, "An experimental study of the effects of drilling solids on frictional pressure losses in coiled tubing", Society of Petroleum Engineers, vol. 67191, pp. 1-15, 2001.

[10] S. Shah, Y. X. Zhou, M. Bailey, and J. Hernandez, "Correlations to predict frictional pressure loss of hydraulic fracturing slurry in coiled tubing", Society of Petroleum Engineers Production \& Operations, vol. 104253, pp. 381-395, 2009.

[11] B. N. Rao, "Friction factor for turbulent flow of non-newtonian fluid in coiled tubing", Society of Petroleum Engineers, vol. 74847, pp. 1-12, 2002.

[12] Y. Zhou, and S. N. Shah, "New friction-factor correlations for nonnewtonian fluid flow in coiled tubing", Society of Petroleum Engineers Drilling \& Completion, vol. 77960, pp. 68-76, 2006.

[13] M. B. Bailey, and R. S. Rosine, "Comparison of computional fluid dynamics of erosion in coiled tubing on reel-to-injector flow area", SPE/ICoTA Coiled Tubing \& Well Intervention Conference and Exhibition, 31 March-1 April 2009, The Woodlands, Texas, 2009.

[14] D. J. Ma, G. S. Li, Z. W. Huang, J. L. Niu, C. Hou, Mi. J. Liu, and J. B. Li, "A model of calculating the circulating pressure loss in coiled tubing ultra-short radius radial drilling", Petroleum Exploration and Development vol. 39, no. 4, pp. 528-533, 2012.

[15] Y. Zhou, "Theoretical and experimental studies of power-law fluid flow in coiled tubing", Oklahoma: The University of Oklahoma, 2006.

[16] T. Niu, H. W. Liang, and Y. J. Xu, "Study on the pressure loss laws in micro hole circulatory system", Drilling \& Production Technology, vol. 32, no.4, pp. 31-33, 2009.

[17] H. L. Zhao, Z. H. Ke, and Z. F. Zhao, "Development and application on slimhole and coiled tubing technology", Beijing: Petroleum Industry Press, pp. 40-41, 1998.

[18] W. R. Dean, "Note on the motion of fluid in a curved pipe", Philosophical Magazine, vol. 04, no. 20, pp. 208-223, 1927.

[19] W. R. Dean, "Fluid Motion in a Curved Channel", Proceedings of the Royal Society of London: Series A, Containing Papers of a 
Mathematical and Physical Character, vol. 121, no. 787, pp. 402420, 1928.

[20] N. Bharath, "Friction Factors for Turbulent Flow of NonNewtonian Fluids in Coiled Tubing", SPE/ICoTA Coiled Tubing Conference and Exhibition, 9-10 April 2002, Houston, Texas, 2002.
[21] X. J. Hou, D. L. Gao, and Z. H. Shen, "An analysis of the flow resistance in coiled tubing wound around a reel, in microhole drilling", CMES - Computer Modeling in Engineering and Sciences, vol. 89, no. 2, pp. 97-109, 2013.

[22] Z. M. Zhao, "Coiled Tubing Engineering Technical Manual", Beijing: Petroleum Industry Press, pp. 71-78, 2011.

Received: May 05, 2013

Revised: December 02, 2013

Accepted: December 10, 2013

(C) Xuejun et al.; Licensee Bentham Open.

This is an open access article licensed under the terms of the Creative Commons Attribution Non-Commercial License (http://creativecommons.org/licenses/by-nc/3.0/) which permits unrestricted, non-commercial use, distribution and reproduction in any medium, provided the work is properly cited. 